\title{
Reliability and Validity of a Questionnaire to Assess Swallowing Disorders in Parkinson's Disease Cases
}

\author{
${ }^{1}$ Unnikrishnan Menon, ${ }^{2}$ Suresh K Radhakrishnan, ${ }^{3} \mathrm{KR}$ Sundaram
}

\begin{abstract}
Introduction: Swallowing problems have significant subjective component. Their evaluation necessitates the use of questionnaires to know both extent of symptoms and effect on quality of life. These should be designed to get maximum information with minimum trouble to the patient. Parkinson's disease (PD) involves swallowing disorder as a symptom, often unreported. Hence, this needs to be assessed separately.
\end{abstract}

Aim: To develop a questionnaire, in the vernacular, to check for the presence of swallowing problems in PD patients and to study its reliability and validity.

Materials and methods: We developed an 11-item questionnaire in English, modeled on the Tel Aviv swallowing disturbance questionnaire (SDQ), chosen for its brevity. This was translated into the vernacular (Malayalam, native tongue of the state of Kerala in India) and administered to patients $(n=106)$ attending the weekly PD clinic of a tertiary care center, in consultation with the attending $\mathrm{n}$ in charge of movement disorder patients. The same questionnaire was also administered to age-matched controls (patients without any swallowing disorders) $(n=63)$ attending the ear, nose, and throat (ENT); neurology; and geriatric clinics. The scores were documented and statistical analysis done, to check for internal reliability and validity.

Results: Comparison of mean total scores between cases (2.89) and controls (1.51) showed a statistically significant difference, indicating statistical validity $(p=0.009)$. Guttman's split-half correlation showed fairly good reliability for both cases (0.812) and controls (0.851).

Conclusion: The present questionnaire to assess dysphagia in PD patients has been found to be reliable and valid for use. We recommend it as a screening tool in the neurology outpatient department (OPD).

\footnotetext{
${ }^{1}$ Associate Professor, ${ }^{2,3}$ Professor

${ }^{1}$ Department of ENT, Amrita Institute of Medical Sciences and Research Centre, Amrita Vishwa Vidyapeetham, Kochi, Kerala India

${ }^{2}$ Department of Neurology, Amrita Institute of Medical Sciences and Research Centre, Amrita Vishwa Vidyapeetham, Kochi Kerala, India

${ }^{3}$ Department of Biostatistics, Amrita Institute of Medical Sciences and Research Centre, Amrita Vishwa Vidyapeetham, Kochi Kerala, India

Corresponding Author: Unnikrishnan Menon, Associate Professor, Department of ENT, Amrita Institute of Medical Sciences and Research Centre, Amrita Vishwa Vidyapeetham Kochi, Kerala, India, Phone: +919447831755, e-mail: unnikrishnanmenon8@gmail.com
}

Keywords: Deglutition, Deglutition disorders, Parkinson's disease, Questionnaire, Reliability and validity.

How to cite this article: Menon U, Radhakrishnan SK, Sundaram KR. Reliability and Validity of a Questionnaire to Assess Swallowing Disorders in Parkinson's Disease Cases. Int J Phonosurg Laryngol 2016;6(2):78-82.

Source of support: Nil

Conflict of interest: None

\section{INTRODUCTION}

Dysphagia, as with any other disease entity, involves detailed history taking and clinical examination as the first steps of evaluation. These, along with relevant investigations, help in the diagnosis of both site and cause of the problem. However, there can be two situations peculiar to dysphagia, which may not be covered by the above. Firstly, the possible lack of awareness, on the part of the patient and/or caregivers, of the extent of the problem. ${ }^{1,2}$ Second is the extent to which the problem has affected the patient's lifestyle. ${ }^{3}$ These two issues are usually addressed by the use of questionnaires, to be answered by the patient and/or caregiver. The former entails a set of questions specifically directed at the different levels of swallowing; the latter, at the aspects of quality of life affected by the problem. These could also vary, depending on the intended patient population and cause of dysphagia. There are many internationally accepted validated questionnaires used consistently for these purposes. ${ }^{4-8}$ Length and technical details often hamper the effectiveness of many standard questionnaires, especially in the Indian setting. The present study is an attempt to use a new questionnaire for use as a screening tool for subjective dysphagia in Parkinson's disease (PD) patients.

\section{MATERIALS AND METHODS}

Type of study: Prospective cross-sectional study. Setting: Tertiary care medical center catering to a population of approximately 30 million in the state of Kerala in Southern India.

The study was planned by the otolaryngologist dealing with swallowing disorders and the neurologist dealing with movement disorders. The idea was to check for swallowing problems perceived by PD patients, attending a weekly PD clinic at the Amrita Institute of 
Medical Sciences and Research Centre. Diagnosis of PD was made on the basis of the United Kingdom Parkinson's Disease Society Brain Bank clinical diagnostic criteria.

Validated questionnaires already in use were considered. However, the main drawback was the number and type of questions, some needing details to be entered by the patient. This had to be done during the waiting period in the clinic by the patients themselves. We felt the need for a simple yet relevant questionnaire that would readily reveal any swallowing problem felt by the patient. The main aspects had to be comprehensibility and the need for minimal writing. The former entailed the use of the vernacular.

In the Indian context, the reliability of questionnaire responses, in general, tends to be suspect. With this in mind, we needed to use a questionnaire which was neither too long nor involved too much of writing. Our focus was on brevity, with Yes/No or "tick the option" type of questions. For the questionnaire to be short, the number of questions had to be kept to the bare minimum. This meant the inclusion of only those questions that pertained to the swallowing disorders likely to present in PD, and the exclusion of associated symptoms and quality of life-related questions.

As a template, we chose the Tel Aviv Sourasky University Swallowing Disturbance Questionnaire (SDQ). ${ }^{8}$ It has 15 questions, with ticking as the only option to be carried out. We narrowed the questions down to 11. This was first made in English; the format is as shown in the Appendix 1.

Next was the issue of translation into the vernacular. Many validated English-language questionnaires have been successfully translated into the vernacular, with the obvious intent of including the maximum number of patients. ${ }^{9,10}$ Hence, our 11-question set was translated into Malayalam, the language spoken by the people of the state of Kerala, where our institution is situated. We already have experience with a validated Malayalam voice handicap index 30, which is one of the standard questionnaires used in voice disorders. ${ }^{11}$ All the questions were in the form of simple, concise statements, so there was no necessity for a medical translator. The translated questionnaire was first administered as a pilot, to a few patients attending the neurology outpatient department (OPD). The responses were satisfactory. We then proceeded to administer it to the study population. This consisted of patients attending the weekly PD clinic in the neurology department. The questionnaire was administered by an assigned staff in the clinic, with instructions to the patient and accompanying caregiver about the method (ticking one of the three options for each question). As far as possible, the patients were encouraged to do this themselves. In case of inability due to fine motor control, the caregiver was allowed to do the physical act of ticking after the patient had read the question and conveyed the answer. Patients who could not read, for whatever reason, were excluded from the study. The questionnaire was administered as a one-time process, so that an individual patient responded only once during the PD clinic visits.

With a view to checking for reliability, the same questionnaire was administered to age-matched controls, consisting of patients visiting the neurology; ear, nose, and throat (ENT); and geriatric OPDs. We ensured that none of these patients had swallowing difficulty as a presenting symptom. For validity, it would have been ideal to administer another standard questionnaire. However, logistical and patient compliance issues precluded this.

The questionnaires were administered over a period of 15 months (October 2014 to December 2015). The responses were tabulated and statistical analysis applied.

\section{RESULTS}

The responses were obtained from 106 PD patients (67 males, 39 females). Mean age was 66 years. Controls were 63 in number, with mean age of 67 years. Comparison of age between cases (mean: 65.9, standard deviation [SD]: 8.6) and control groups (mean: 67.2, SD: 4.4) showed the two groups to be comparable with respect to age (Table 1 ).

The scoring pattern for the questionnaire meant that there could be a minimum score of 0 , indicating no subjective swallowing difficulty whatsoever, to a maximum of 22.

The parameters that we studied for the purpose of this study were the comparison between responses of cases and controls, and correlation between each question and total score.

For the cases, the mean response was 2.89 with SD of 3.99. For the controls, the corresponding values were 1.51 and 2.76; p-value was 0.009 , indicating a statistically significant difference between cases and controls. This showed statistical validity with respect to the total score (Table 2).

Next, we checked the statistical correlation of individual question response score with the total score. For the cases, the correlation coefficient values ranged from 0.47 for question 6 to 0.72 for question 2. Guttman's split-half

Table 1: Comparison of age between cases and control groups

\begin{tabular}{llllll}
\hline & Group & $n$ & Mean & $S D$ & $p$-value \\
\hline \multirow{2}{*}{ Age } & Control & 63 & 67.21 & 4.422 & 0.194 \\
& Cases & 106 & 65.90 & 8.616 & \\
\hline
\end{tabular}

The results indicate that the two groups are comparable with respect to age 
Table 2: Comparison of mean total scores between cases and control groups

\begin{tabular}{llllll}
\hline & Group & $n$ & Mean & $S D$ & $p$-value \\
\hline Total & Control & 63 & 1.51 & 2.758 & 0.009 \\
& Cases & 106 & 2.89 & 3.994 & \\
\hline
\end{tabular}

The results show a statistically significant difference indicating statistical validity with respect to the total score

correlation was found to be 0.812 , indicating a fairly good reliability (Table 3). Similar results were noted with the scores of the controls (Table 4).

For further clarity, we compared cases and controls for each question, by applying Wilcoxon's rank sum test. It was found that the difference, on an average, was statistically significant for questions $1,2,3,6$, and 10, indicating statistical validity for these questions (Table 5 ).

\section{DISCUSSION}

Subjective evaluation of a symptom is an integral aspect of many disease conditions. ${ }^{12}$ The internationally followed method for this is by using questionnaires to be answered by the patient. The construction of such questionnaires is often a balancing act between comprehensive coverage of the issues, from the medical point of view, and comprehension and ease of answering on the patient's part. ${ }^{13}$ In the Indian setting, the latter aspect has to be particularly carefully addressed as linguistic and cultural issues create hurdles. A reliable, validated questionnaire used internationally, in English, for a particular disease, may not be suitable to cover the patient population of that disease in a non-English native speaking group. This is often applicable to many countries, apart from

Table 3: Percentage distribution of responses and mean and SD for each question and the correlation between each question with total score and age of the cases

\begin{tabular}{|c|c|c|c|c|c|c|c|}
\hline \multirow[b]{2}{*}{ Questions } & \multicolumn{3}{|c|}{ Percentages } & \multirow[b]{2}{*}{ Mean } & \multirow[b]{2}{*}{$S D$} & \multicolumn{2}{|c|}{ Spearman rank correlation } \\
\hline & 0 & 1 & 2 & & & With total score (r1) & With age (r2) \\
\hline \multicolumn{8}{|l|}{ Cases } \\
\hline Q1 & 65.09 & 22.64 & 12.26 & 0.47 & 0.71 & 0.71 & 0.20 \\
\hline Q2 & 72.64 & 20.75 & 6.60 & 0.34 & 0.60 & 0.72 & 0.22 \\
\hline Q3 & 74.53 & 15.09 & 10.38 & 0.36 & 0.66 & 0.59 & 0.34 \\
\hline Q4 & 77.36 & 17.92 & 4.72 & 0.27 & 0.54 & 0.66 & 0.25 \\
\hline Q5 & 77.36 & 16.04 & 6.60 & 0.29 & 0.59 & 0.65 & 0.11 \\
\hline Q6 & 86.79 & 13.21 & 0 & 0.13 & 0.34 & 0.47 & 0.21 \\
\hline Q7 & 82.08 & 15.09 & 2.83 & 0.21 & 0.47 & 0.58 & 0.13 \\
\hline Q8 & 83.96 & 11.32 & 4.72 & 0.21 & 0.51 & 0.56 & 0.32 \\
\hline Q9 & 77.36 & 21.70 & 0.94 & 0.24 & 0.45 & 0.63 & 0.17 \\
\hline Q10 & 81.13 & 16.98 & 1.89 & 0.21 & 0.45 & 0.57 & 0.33 \\
\hline Q11 & 84.91 & 15.09 & 0 & 0.15 & 0.36 & 0.54 & 0.22 \\
\hline Total & & & & 2.89 & 3.99 & & 0.27 \\
\hline
\end{tabular}

Correlation coefficient values ranged from 0.47 for question 6 to 0.72 for question 2. Guttman's split-half correlation was found to be 0.812 , indicating a fairly good reliability

Table 4: Percentage distribution of responses and mean and SD for each question and the correlation between each question with total score and age of the controls

\begin{tabular}{|c|c|c|c|c|c|c|c|}
\hline \multirow[b]{2}{*}{ Questions } & \multicolumn{3}{|c|}{ Percentages } & \multirow[b]{2}{*}{ Mean } & \multirow[b]{2}{*}{$S D$} & \multicolumn{2}{|c|}{ Spearman rank correlation } \\
\hline & 0 & 1 & 2 & & & With total score (r1) & With age (r2) \\
\hline \multicolumn{8}{|l|}{ Controls } \\
\hline Q1 & 80.95 & 12.70 & 6.35 & 0.25 & 0.57 & 0.69 & 0.19 \\
\hline Q2 & 85.71 & 14.29 & 0 & 0.14 & 0.35 & 0.65 & 0.05 \\
\hline Q3 & 88.89 & 11.11 & 0 & 0.11 & 0.32 & 0.54 & -0.06 \\
\hline Q4 & 85.71 & 12.70 & 1.59 & 0.16 & 0.41 & 0.57 & -0.05 \\
\hline Q5 & 85.71 & 12.70 & 1.59 & 0.16 & 0.41 & 0.59 & 0.15 \\
\hline Q6 & 95.24 & 4.76 & 0 & 0.05 & 0.21 & 0.40 & 0.07 \\
\hline Q7 & 88.89 & 11.11 & 0 & 0.11 & 0.32 & 0.49 & 0.07 \\
\hline Q8 & 90.48 & 7.94 & 1.59 & 0.11 & 0.36 & 0.55 & 0.23 \\
\hline Q9 & 80.95 & 19.05 & 0 & 0.19 & 0.40 & 0.65 & 0.18 \\
\hline Q10 & 95.24 & 4.76 & 0 & 0.05 & 0.21 & 0.38 & -0.10 \\
\hline Q11 & 84.13 & 15.87 & 0 & 0.16 & 0.37 & 0.64 & 0.16 \\
\hline Total & & & & 1.51 & 2.76 & & 0.13 \\
\hline
\end{tabular}

Correlation coefficient values ranged from 0.38 for question 10 to 0.69 for question 1 . Guttman's split-half correlation was found to be 0.851 , indicating a fairly good reliability 
Table 5: Comparison between cases and controls for each question

\begin{tabular}{lllllll}
\hline & \multicolumn{2}{c}{ Cases (106) } & & \multicolumn{2}{c}{ Control (63) } & \\
\cline { 2 - 3 } \cline { 5 - 6 } Questions & Mean & $S D$ & & Mean & $S D$ & p-value \\
\hline Q1 & 0.47 & 0.71 & & 0.25 & 0.57 & 0.028 \\
Q2 & 0.34 & 0.60 & & 0.14 & 0.35 & 0.05 \\
Q3 & 0.36 & 0.66 & & 0.11 & 0.32 & 0.025 \\
Q4 & 0.27 & 0.54 & & 0.16 & 0.41 & 0.186 \\
Q5 & 0.29 & 0.59 & & 0.16 & 0.41 & 0.186 \\
Q6 & 0.13 & 0.34 & & 0.05 & 0.21 & 0.078 \\
Q7 & 0.21 & 0.47 & & 0.11 & 0.32 & 0.237 \\
Q8 & 0.21 & 0.51 & & 0.11 & 0.36 & 0.234 \\
Q9 & 0.24 & 0.45 & & 0.19 & 0.40 & 0.582 \\
Q10 & 0.21 & 0.45 & & 0.05 & 0.21 & 0.01 \\
Q11 & 0.15 & 0.36 & & 0.16 & 0.37 & 0.978 \\
\hline
\end{tabular}

On applying Wilcoxon's rank sum test, it was found that the difference, on an average, was statistically significant for questions $1,2,3,6$, and 10 (borderline significance), indicating statistical validity for these questions in the questionnaire

India. This is usually resolved by simply translating and validating an existing questionnaire. The other issue often encountered among Indian patient groups is the lack of awareness of the relevance of such questionnaires in the overall disease management plan. This leads to lack of willingness or inadequate responses. Although there are no studies or documentation of this particular aspect, it is experienced or discussed as an anecdotal fact, among health care professionals. Hence, the specific challenge of making an effective, useful questionnaire for Indian populations in the vernacular.

With a common interest in the field of swallowing disorders in neurological conditions, the chief authors planned to construct a questionnaire for the same. Considering the volume of patients in our institutional setting, it was decided to do this specifically for PD. This is a disorder with predominantly motor symptoms. Nonmotor symptoms like dysphagia are given less importance in the overall perception and assessment of the disease. Even in the widely used Unified Parkinson's Disease Rating Scale for assessment of PD patients, dysphagia assessment is limited to only a few questions. It is believed that symptoms like dysphagia are not dopamine dependent and hence may not respond very well to the usual therapies available for PD.

Dysphagia is generally believed to be a late symptom in PD. However, in some patients, it may be of early onset. The challenge for the medical personnel and caretakers lies in detecting the exact onset of the problem and addressing the subsequent quality of life issues. Literature search revealed a variety of questionnaires for dysphagia in general. However, the issues referred to earlier were felt to be stumbling blocks. Hence, a reasonably short, symptom-specific questionnaire was prepared. It covered almost all the issues usually encountered in the spectrum of swallowing disorders, but with stress on the oral ones. This stage is known to be the most affected in PD. ${ }^{14}$ The first step was to check its reliability and validity.

The results have been reasonably satisfying, in terms of overall reliability and validity. So, we propose the use of this tool in PD patients. It can also potentially be used as a screening tool for all neurology patients who are likely to develop swallowing issues as part of their disease process.

The one area of concern in the present questionnaire is the reduced statistical validity for some of the questions. On analyzing, these were the questions concerning pharyngeal and laryngeal stages of swallowing. It may be assumed that these would show validity in other conditions, especially stroke. The other assumption would be to consider the further editing of this questionnaire to use only the statistically valid questions for PD patients.

As a next step, the authors plan to create cut-off values for the score from this questionnaire administered to more PD patients. This could then be specifically used as an early screening tool and to decide the need for dysphagia-specific investigations in PD, such as videofluoroscopy.

\section{CONCLUSION AND RECOMMENDATIONS}

- The present 11-question tool to assess dysphagia has been studied and found to be reliable and valid for use in PD patients.

- This questionnaire can be used as a quick screening tool in the neurology outpatient setting to check for subjective swallowing disorders.

- Modifications of this questionnaire can be used, in Indian populations, targeted for disease entities known to cause dysphagia.

\section{ACKNOWLEDGMENT}

Authors would like to thank Ms Shikha, MSW in Neurology Department, for help in data collection. The author also acknowledges the help given by Dr Urmi Bapat in manuscript corrections.

\section{REFERENCES}

1. Becker DLS. Patient awareness of dysphagia [dissertation]. University of Iowa; 2011.

2. Kalf JG, De Swart BJM, Bloem BR, Munneke M. Prevalence of oropharyngeal dysphagia in Parkinson's disease: a meta-analysis. Parkinsonism Relat Disord 2012 May;18(4): 311-315.

3. Eslick GD, Talley NJ. Dysphagia: epidemiology, risk factors and impact on quality of life-a population-based study. Aliment Pharmacol Ther 2008 May;27(10):971-979. 
4. Chen AY, Frankowski R, Bishop-Leone J, Hebert T, Leyk S, Lewin J, Goepfert H. The development and validation of a dysphagia-specific quality-of-life questionnaire for patients with head and neck cancer: the MD Anderson dysphagia inventory. Arch Otolaryngol Head Neck Surg 2001 Jul;127(7):870-876.

5. Dysphagia Questionnaire. Baylor All Saints Medical Centres. p. 1-4.

6. Northwestern Dysphagia Patient Check Sheet. p. 1-4. Available from: www.AmericanDysphagiaNetwork.org.

7. SWAL-QOL Survey. p. 1-11.

8. Manor Y, Giladi N, Cohen A, Fliss DM, Cohen JT. Validation of a swallowing disturbance questionnaire for detecting dysphagia in patients with Parkinson's disease. Mov Disord 2007 Oct 15;22(13):1917-1921.

9. Becker AE, Thomas JJ, Bainivualiku A, Richards L, Navara K, Roberts AL, Gilman SE, Striegel-Moore RH. Validity and reliability of a Fijian translation and adaptation of the Eating Disorder Examination Questionnaire. Int J Eat Disord 2010 Mar;43(2):171-178.
10. Rajaei A, Azargoon SA, Nilforoush MH, Barzegar Bafrooei E, Ashtari F, Chitsaz A. Validation of the Persian translation of the swallowing disturbance questionnaire in Parkinson's disease patients. Parkinsons Dis 2014;2014:159476.

11. Menon U, Sheejamol VS, Cherian MP. Validation of Malayalam version of the voice handicap index. Int J Phonosurg Laryngol 2012;2(1):1-4

12. Muldoon MF, Barger SD, Flory JD, Manuck SB. What are quality of life measurements measuring? BMJ 1998 Feb;316(7130):542-545.

13. Subar AF, Ziegler RG, Thompson FE, Johnson CC, Weissfeld JL, Reding D, Kavounis KH, Hayes RB, Prostate, Lung, Colorectal, and Ovarian Cancer Screening Trial Investigators. Is shorter always better? Relative importance of questionnaire length and cognitive ease on response rates and data quality for two dietary questionnaires. Am J Epidemiol 2001 Feb 15;153(4):404-409.

14. Logemann JA. Swallowing problems associated with degenerative disease. In: Berman D, editor. Evaluation and treatment of swallowing disorders. 2nd ed. Austin (TX): Pro.Ed; 1998. p. 334-335.

\section{APPENDIX 1: Questionnaire on swallowing difficulties}

\section{Name:}

MRD No.:

1. Does it take more time or effort to chew a food bolus?

- Never

- Sometimes

- Always

2. Does the food bolus get stuck in the mouth after chewing?

- Never

- Sometimes

- Always

3. Do food particles or water drops spill out of your mouth during or after chewing?

- Never

- Sometimes

- Always

4. Does food bolus remain in your mouth even after swallowing?

- Never

- Sometimes

- Always

5. Do you feel difficulty in initiating a swallowing?

- Never

- Sometimes

- Always

6. Does food bolus or water get into your nose during or just after a swallow?

- Never

- Sometimes

- Always

\section{Age:}

PD Clinic No.:

Sex:

7. Do food particles remain sticking in your throat after completion of a swallow?

- Never

- Sometimes

- Always

8. Do you need more than two swallows to clear a single bolus from your throat?

- Never

- Sometimes

- Always

9. Have you experienced choking sensation or cough at the time of swallow?

- Never

- Sometimes

- Always

10. Have you noticed any change in voice just after a swallow?

- Never

- Sometimes

- Always

11. Have you experienced choking sensation or cough after the completion of a swallow?

- Never

- Sometimes

- Always

- Never-0

- Sometimes-1

- Always - 2 